\title{
Stochastic resonance between dissipative structures in a bistable noise-sustained dynamics
}

\author{
B. von Haeften, ${ }^{1}$ G. Izús, ${ }^{1}$ S. Mangioni, ${ }^{1}$ A. D. Sánchez, ${ }^{1}$ and H. S. Wio ${ }^{2,3, *}$ \\ ${ }^{1}$ Departamento de Física, FCEyN, Universidad Nacional de Mar del Plata, Deán Funes 3350, 7600 Mar del Plata, Argentine \\ ${ }^{2}$ Grupo de Física Estadística, Centro Atómico Bariloche and Instituto Balseiro, 8400 San Carlos de Bariloche, Argentine \\ ${ }^{3}$ Departament de Física, Universitat de les Illes Balears and IMEDEA, E-07122 Palma de Mallorca, Spain
}

(Received 9 September 2003; published 23 February 2004)

\begin{abstract}
We study an extended system that without noise shows a monostable dynamics, but when submitted to an adequate multiplicative noise, an effective bistable dynamics arises. The stochastic resonance between the attractors of the noise-sustained dynamics is investigated theoretically in terms of a two-state approximation. The knowledge of the exact nonequilibrium potential allows us to obtain the output signal-to-noise ratio. Its maximum is predicted in the symmetric case for which both attractors have the same nonequilibrium potential value.
\end{abstract}

DOI: 10.1103/PhysRevE.69.021107

\section{INTRODUCTION}

During the last few decades a wealth of research results on fluctuations or noise have led us to the recognition that in many situations noise can actually play a constructive role that induces new ordering phenomena. Some examples are stochastic resonance in zero-dimensional and extended systems [1-6], noise-induced transitions [7], noise-induced phase transitions [8,9], noise-induced transport [10-13], noise-sustained patterns [14-16], noise-induced limit cycle [17], etc.

The phenomenon of stochastic resonance (SR) - namely, the enhancement of the output signal-to-noise ratio (SNR) caused by injection of an optimal amount of noise into a nonlinear system-stands as a puzzling and promising cooperative effect arising from the interplay between deterministic and random dynamics in a nonlinear system. The broad range of phenomena-drawn from almost every scientific endeavor-for which this mechanism can offer an explanation has been put in evidence by many reviews and conference proceedings. See Ref. [1] and references there to scan the state of the art.

Most of the phenomena that could possibly be explained by SR occur in extended systems: for example, diverse experiments were carried out to explore the role of SR in sensory and other biological functions [18] or in chemical systems [19]. These were, together with the possible technological applications, the motivation to many recent studies showing the possibility of achieving an enhancement of the system response by means of the coupling of several units in what conforms an extended medium $[2,3,5,6,20]$, or analyzing the possibility of making the system response less dependent on a fine-tuning of the noise intensity, as well as different ways to control the phenomenon [21,22].

In some previous papers [3-6] we have studied the stochastic resonant phenomenon in extended systems for the transition between two different patterns, and exploited the concept of nonequilibrium potential $[23,24]$. The nonequilibrium potential is a special Lyapunov functional of the associated deterministic system which for nonequilibrium sys-

*Electronic address: wio@cab.cnea.gov.ar
PACS number(s): 05.40.Ca, 05.45.- a, 82.40.Ck

tems play a role similar to that played by a thermodynamic potential in equilibrium thermodynamics [23]. Such a nonequilibrium potential, closely related to the solution of the time independent Fokker-Planck equation of the system, characterizes the global properties of the dynamics: that is attractors, relative (or nonlinear) stability of these attractors, and height of the barriers separating attraction basins, and in addition it allows us to evaluate the transition rates among the different attractors.

In this work we analyze a new aspect of such a problem studying SR between the attractors of the noise-sustained dynamics $[14,15]$, that is: the same noise source that induces the dynamics induces the transitions among both structures, and produces the stochastic resonant phenomenon. Some closely related work corresponds to the so-called doubly stochastic resonance [25], as well as to another previous work [26] related to noise-induced phase transitions $[8,9]$. In both cases the authors have mainly resorted to a standard meanfield approach, or to an estimate of the effective potential, while here we obtain the exact form of the noise-induced patterns (stable and unstable ones) as well as the complete form of the nonequilibrium potential. In this way we can obtain the transition rates and clearly quantify the SR phenomenon by means of the SNR.

The organization of the paper is as follows. In Sec. II we present the model and formalism to be used. After that, we discuss in Sec. III the stochastic resonance phenomenon between the homogeneous structure and the inhomogeneous pattern. Finally, we present in Sec. IV some conclusions and future perspectives.

\section{THE MODEL}

We consider a one-dimensional system, limited to the region $-L / 2 \leqslant x \leqslant L / 2$, described by the following deterministic equation:

$$
\partial_{t} \phi(x, t)=\partial_{x}\left[D(\phi) \partial_{x} \phi\right]+F(\phi),
$$

assuming Dirichlet boundary conditions [that is $\phi( \pm L / 2)$ $=0]$. This equation can be written in a variational form as

$$
\partial_{t} \phi(x, t)=-\frac{1}{D(\phi)} \frac{\delta V[\phi]}{\delta \phi(x)},
$$


where the potential

$V[\phi]=\int_{-L / 2}^{L / 2} d x\left\{-\int_{0}^{\phi} d \phi^{\prime} D\left(\phi^{\prime}\right) F\left(\phi^{\prime}\right)+\frac{1}{2}\left[D(\phi) \partial_{x} \phi\right]^{2}\right\}$

is a Lyapunov functional [while $D(\phi)>0$ ] for the deterministic dynamics and it is essentially the logarithm of the probability density of configuration when Eq. (1) is perturbed by an additive source of spatiotemporal white noise.

The starting point of our stochastic analysis will be Eq. (1) with an additional multiplicative noise, in the Stratonovich interpretation, given by

$$
\partial_{t} \phi(x, t)=-\frac{1}{D(\phi)} \frac{\delta V[\phi]}{\delta \phi(x)}+g(\phi) \xi(x, t),
$$

where $\xi$ is a Gaussian noise with zero mean and correlation $\left\langle\xi(x, t) \xi\left(x^{\prime}, t^{\prime}\right)\right\rangle=2 \epsilon \delta\left(x-x^{\prime}\right) \delta\left(t-t^{\prime}\right), \epsilon$ being the noise intensity. For the coefficient of the noise term, $g(\phi)$, we adopt

$$
g(\phi)=\frac{1}{\sqrt{D(\phi)}}
$$

in order to guarantee that the fluctuation-dissipation relation is fulfilled [27].

As we are considering the Stratonovich interpretation, the stationary solution of the associated Fokker-Planck equation can be written as [28]

$$
P_{s t}[\phi] \sim \exp \left(-V_{\text {eff }} / \epsilon\right)
$$

where the effective potential $V_{\text {eff }}[\phi]$ is given by

$$
V_{\mathrm{eff}}[\phi]=V[\phi]-\lambda \int_{-L / 2}^{L / 2} d x \ln D(\phi)
$$

Here $\lambda$ is a renormalized parameter related to $\epsilon$ through $\lambda$ $=\epsilon /(2 \Delta x)$ in a lattice discretization, where $\Delta x$ is the lattice parameter [28].

The extremes of $V_{\text {eff }}$ correspond to the stationary fixed points of the noise-sustained dynamics. They can be computed from the first variation of $V_{\text {eff }}(\phi)$ with respect to $\phi$ equal to zero, that is,

$$
\begin{aligned}
\delta V_{\mathrm{eff}}\left[\phi_{s t}\right]= & -\int_{-L / 2}^{L / 2} D(\phi)\left[\partial_{x}\left(D(\phi) \partial_{x} \phi\right)\right. \\
& \left.+F_{\mathrm{eff}}(\phi)\right]\left.\delta \phi(x) d x\right|_{\phi=\phi_{s t}}=0,
\end{aligned}
$$

where

$$
F_{\mathrm{eff}}(\phi)=F(\phi)+\lambda \frac{1}{D(\phi)^{2}} \frac{d}{d \phi} D(\phi)
$$

is the effective nonlinearity which drives the dynamics.

We consider the case of a monostable dynamics in absence of noise

$$
F=-\phi^{3}+b \phi^{2}
$$

and we adopt a model of field-dependent diffusivity which induces an effective bistable dynamics. In particular we have chosen

$$
D(\phi)=\frac{D_{0}}{1+h \phi^{2}}
$$

$\left(D_{0}, h>0\right)$, which corresponds to having a larger diffusivity in low density (low $\phi$ ) regions and a lower diffusivity in high density (large $\phi$ ) ones. With this functional form, $F_{\text {eff }}(\phi)$ in Eq. (9) results in

$$
F_{\mathrm{eff}}=-\phi^{3}+b \phi^{2}-\frac{2 \lambda h \phi}{D_{0}}=\phi\left(\phi-\phi_{1}\right)\left(\phi_{2}-\phi\right),
$$

where $\phi_{1,2}$ depend on parameters, in particular on the control parameter $\lambda$. It is worth noting here that in the deterministic problem $(\lambda=0)$ the reaction term is monostable while, as we increase the noise intensity, the effective nonlinear term $F_{\text {eff }}$ becomes bistable [within the interval $0<\lambda<b^{2} D_{0} /(8 h)$ ] and finally, for $\lambda>b^{2} D_{0} /(8 h)$, becomes again monostable (reentrance effect). Our choice of $F$ and $D$ is one among plenty of different forms for the diffusivity leading to a transition from monostable to bistable and inducing the SR phenomenon [see, for instance, the one used in [28] that corresponds exactly to the inverse of the present diffusion coefficient, i.e., $\left.D(\phi)=D_{0}\left(1+h \phi^{2}\right)\right]$. Density-dependent diffusivities arise in a large variety of systems modeled by reaction-diffusion equations [29]. In biology, for instance, population dynamics is usually driven by a diffusivity that depends on the local population [30]. We can also find examples in physics, a couple of them are in polymer physics (where the diffusion can abruptly drop several orders of magnitude at the gelation point [31]) and in diffusion of hydrogen in metals [32].

A remarkable point is that $\phi=0$ is always a root of $F_{\text {eff }}$ $=0$ (see Fig. 1). This implies [from Eq. (8)] that $\phi(x) \equiv 0$ is an extremum of $V_{\text {eff }}[\phi]$ for all values of $\lambda$. In what follows we will call this structure $\phi_{0}$.

In order to obtain the nonuniform extremes of the potential (and also of the probability density) we must (numerically) solve

$$
\frac{d}{d x}\left(D\left(\phi_{s t}\right) \frac{d}{d x} \phi_{s t}\right)+F_{\mathrm{eff}}\left(\phi_{s t}\right)=0
$$

for the stationary regimen profiles $\phi_{s t}(x)$. This approach allows us to find both the stable and unstable solutions. To analyze their stability we need to calculate $\delta^{2} V_{\text {eff }}$, that defines a Sturm-Liouville problem, with orthogonality weight $D\left(\phi_{s t}\right)$. From that analysis it results that $\phi_{0}$ (defined before) is stable for $\lambda>0$, and in the bistability region we have two nonhomogeneous symmetric patterns: one unstable $\phi_{u}$ (saddle) and one stable $\phi_{s}$. The typical form of these patterns is illustrated in Fig. 2. 


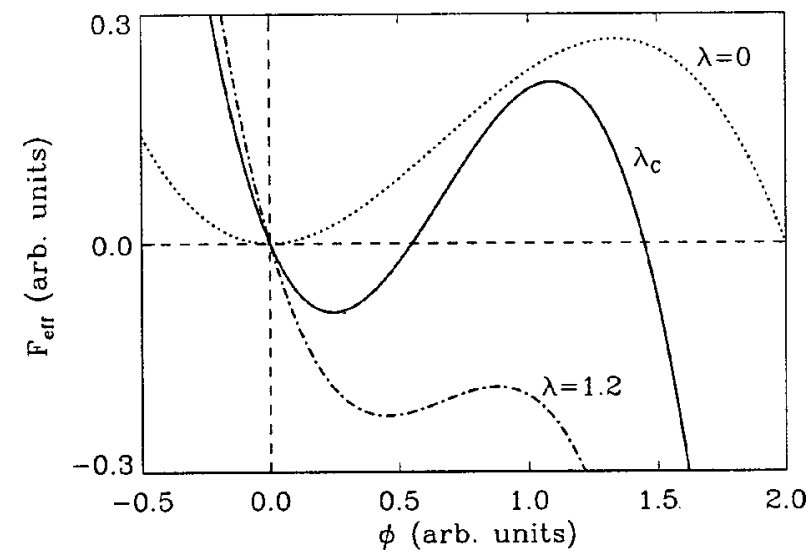

FIG. 1. Form of the nonlinearities for the deterministic case $(\lambda=0)$, bistable case $\left(\lambda=\lambda_{c} \approx 0.8\right)$ and a monostable case $(\lambda$ $=1.2)$ in the reentrance region. The vertical scale was changed in the deterministic case in order to clarify the figure. The parameters used are: $D_{0}=1, h=1 / 2$, and $b=2$. Note that $\phi=0$ remains as a root in all cases.

In Fig. 3 we show $V_{\text {eff }}\left[\phi_{s t}\right]$ vs $\lambda$, evaluated on the different stationary solutions. We define $\lambda_{c}$ as the value of $\lambda$ at which we have symmetrical stability, i.e., where $V_{\text {eff }}\left[\phi_{0}\right]=V_{\text {eff }}\left[\phi_{s}\right]$.

\section{STOCHASTIC RESONANCE BETWEEN STRUCTURES}

We are interested in the stochastic resonance phenomena occurring in the above-described system. For a window of noise intensity the effective dynamics of the system is bistable, corresponding to a noise-induced nontrivial dynamics. We will resort to the so-called two-state approximation [33], all details about the procedure and the evaluation of the SNR could be found in [5]. We consider now that the system is subject, in the adiabatic limit, to a time periodic signal of the form $b=b_{0}+S(t)$ where $S(t)=\Delta b \sin \left(\omega_{0} t\right)$. The usual way of rocking the potential is to introduce an additive periodic forcing (or linear periodic contribution to the potential). However, in the present case, a small periodic variation of $b$

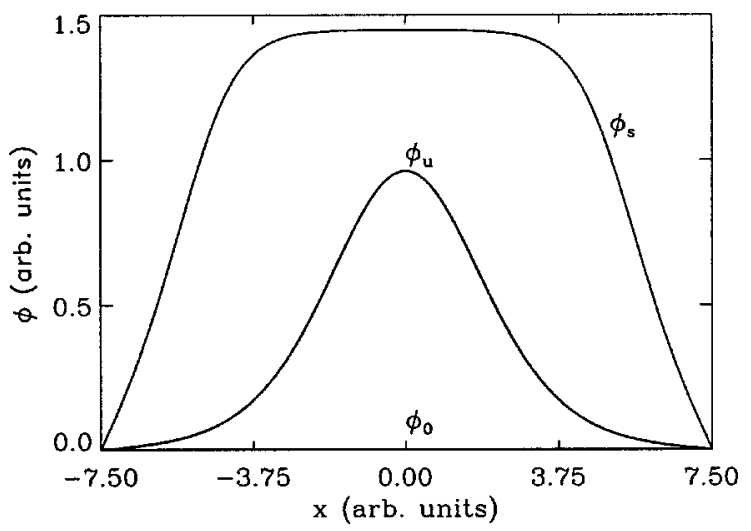

FIG. 2. Fixed points of the noise-sustained dynamics of the problem. We show $\phi_{0} \equiv 0$, the stable homogeneous solution, and both nonhomogeneous patterns: the unstable (saddle) $\phi_{u}$ and the stable one $\phi_{s}$. Here we have $\lambda=\lambda_{c} \approx 0.8$, while the other parameters values are the same as in Fig. 1.

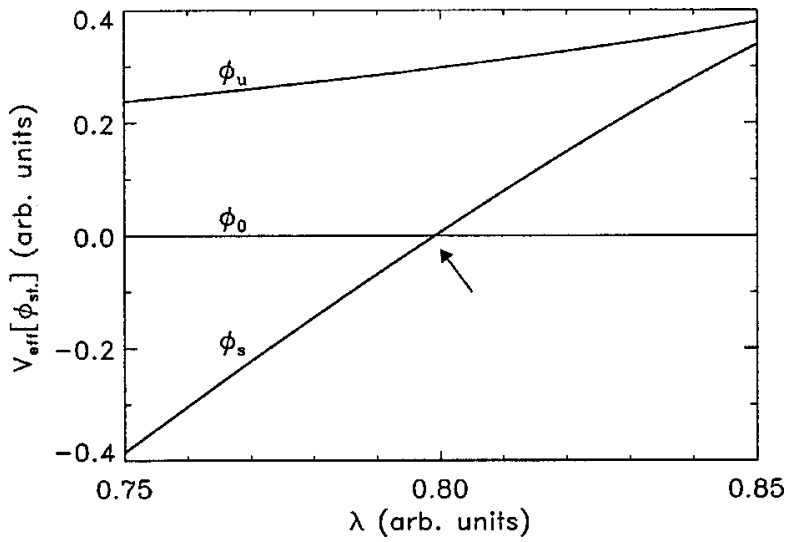

FIG. 3. Nonequilibrium potential $V_{\text {eff }}\left[\phi_{s t}\right]$, as a function of $\lambda$, evaluated on the stationary patterns: curves correspond to stable $\left(\phi_{s}\right)$, homogeneous $\left(\phi_{0}\right)$, and unstable $\left(\phi_{u}\right)$ patterns. The arrow indicates the point where $V_{\text {eff }}\left[\phi_{0}\right]=V_{\text {eff }}\left[\phi_{s}\right]$, corresponding to $\lambda$ $=\lambda_{c} \approx 0.8$.

around $b_{0}$ results to be more sensitive to induce the periodic change in the relative stability of the two attractors.

Up to first-order in the amplitude $\Delta b$ (assumed to be small in order to have a subthreshold periodic input) the transition rates $W_{i}$ take the form

$$
\begin{aligned}
& W_{1}(t)=\mu_{1}-\alpha_{1} \Delta b \sin \left(\omega_{0} t\right), \\
& W_{2}(t)=\mu_{2}+\alpha_{2} \Delta b \sin \left(\omega_{0} t\right),
\end{aligned}
$$

where the constants $\mu_{1,2}$ and $\alpha_{1,2}$ are obtained from the Kramers-like formula for the transition rate [34]

$$
\begin{aligned}
W_{\phi_{i} \rightarrow \phi_{j}}= & \frac{\lambda_{+}}{2 \pi}\left[\frac{\operatorname{det} V_{\text {eff }}\left[\phi_{i}\right]}{\left|\operatorname{det} V_{\text {eff }}\left[\phi_{u}\right]\right|}\right]^{1 / 2} \exp \left[-\left(V_{\text {eff }}\left[\phi_{u}\right]\right.\right. \\
& \left.\left.-V_{\text {eff }}\left[\phi_{i}\right]\right) / \epsilon\right] .
\end{aligned}
$$

Here $\lambda_{+}$is the unstable eigenvalue of the deterministic flux at the relevant saddle point $\left(\phi_{u}\right)$ and

$$
\begin{gathered}
\mu_{1,2}=\left.W_{1,2}\right|_{S(t)=0}, \\
\alpha_{1,2}=\left.\mp \frac{d W_{1,2}}{d S(t)}\right|_{S(t)=0} .
\end{gathered}
$$

These results allows us to calculate the autocorrelation function, the power spectrum, and finally the SNR, that we indicate by $R$. The details of the calculation were shown in Ref. [5]. For $R$, and up to the relevant (second) order in the signal amplitude $\Delta b$, we obtain

$$
R=\frac{\pi}{4 \mu_{1} \mu_{2}} \frac{\left(\alpha_{2} \mu_{1}+\alpha_{1} \mu_{2}\right)^{2}}{\mu_{1}+\mu_{2}}=\frac{\pi}{4 \epsilon} \frac{\mu_{1} \mu_{2}}{\mu_{1}+\mu_{2}} \Phi,
$$

where

$$
\Phi=\int_{-L / 2}^{L / 2} d x \int_{\phi_{0}}^{\phi_{s}(x)} D\left(\phi^{\prime}\right) \phi^{\prime 2} d \phi^{\prime}
$$

gives a measure of the spatial coupling strength. In our case $\phi_{0} \equiv 0$ and 


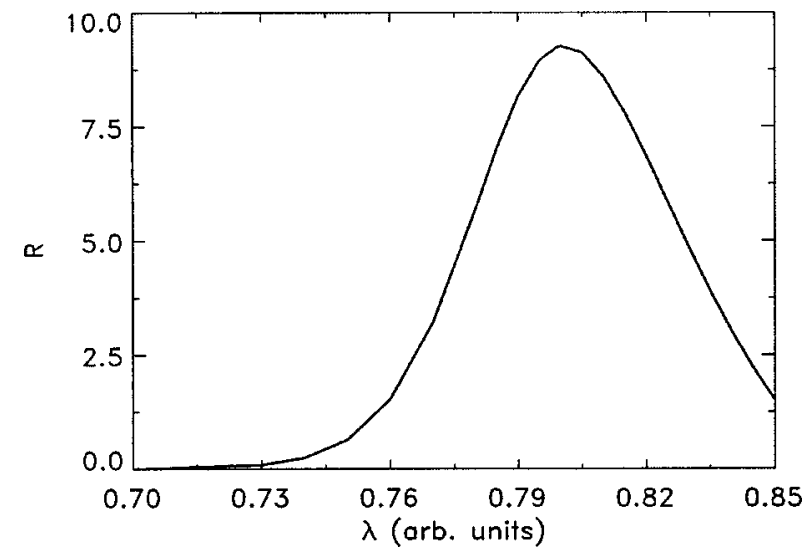

FIG. 4. Signal-to-noise ratio vs $\lambda$ as results from Eq. (17). Here $b_{0}=2$, while other parameters remain unchanged.

$$
\Phi=D_{0} \int_{-L / 2}^{L / 2} d x\left\{\frac{\phi_{s}(x)}{h}-\frac{\arctan \left[\sqrt{h} \phi_{s}(x)\right]}{h^{3 / 2}}\right\} .
$$

In Fig. 4 we show the SNR as a function of the parameter $\lambda$ (which is proportional to $\epsilon$ ). The existence of the typical maximum is the characteristic fingerprint of SR. For a window of noise intensity values, the system enhances the output to the input periodic signal. We see that the maximum SNR occurs at the symmetric situation, that is at $\lambda=\lambda_{c}$.

A similar behavior is observed in general for a wide range of values for $h$ and $D_{0}$ compatible with a bistable effective dynamics. In particular, $\lambda_{c}$ is a monotonically decreasing function of $h$, as we show in Fig. 5. For a given value of $h$, a numerical analysis of Eq. (17) indicates that the maximum of SNR take place at $\lambda_{c}(h)$. Note that, for a given value of $\lambda$, $h$ appears as an additional control parameter that allows a fine-tuning of the symmetrical condition. Finally, in Fig. 6 we show $R_{c}=R\left(\lambda_{c}\right)$ vs $h$ in the range of values where Kramer's formulas apply [35].

\section{CONCLUSIONS}

The study of SR in extended or coupled systems, motivated by both some experimental results and the technological interest, has recently attracted considerable attention $[2-6,20]$. In some previous papers [3-6] we have studied the

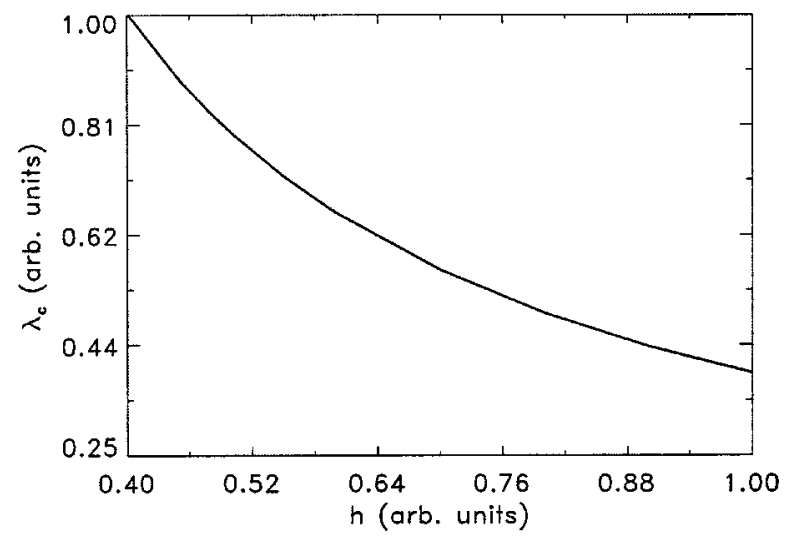

FIG. 5. $\lambda_{c}$ vs $h$ parameter of diffusivity. For small $h$ values $\lambda_{c}$, and hence the noise intensity, increase monotonically.

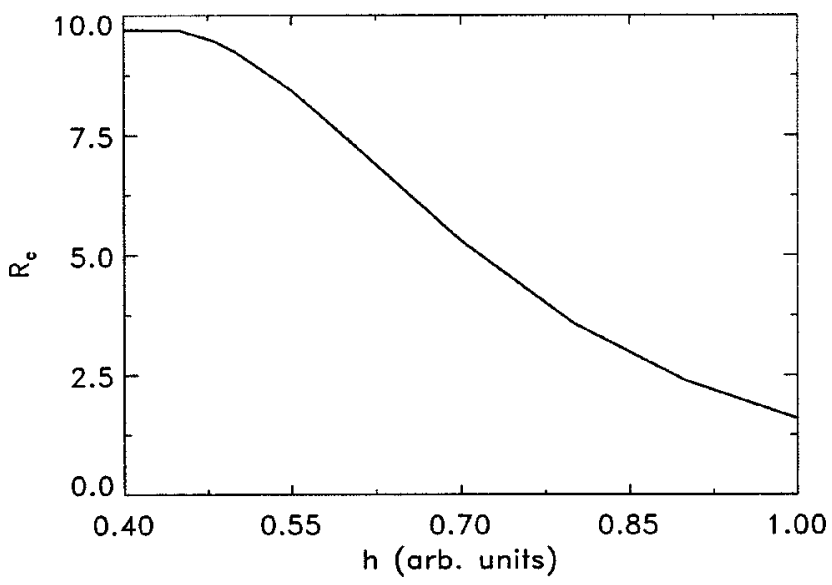

FIG. 6. Signal-to-noise ratio at $\lambda_{c}$ vs $h$. We can see a saturation phenomena as $h$ decreases.

SR phenomenon for the transition between two different patterns, exploiting the concept of nonequilibrium potential $[23,24]$. In this work we have analyzed the SR phenomenon in an extended system from a different point of view, that is, studying SR between two attractors of the noise-sustained dynamics $[14,15]$.

Some closely related work corresponds to the so-called doubly stochastic resonance [25], as well as to a previous work [26] that is tightly related to noise-induced phase transitions $[8,9]$. In both cases the authors have mainly resorted to a standard mean-field approach, or to some estimate of the effective potential. Here we adopt a different approach, obtaining numerically the exact form of the patterns (both the stable and unstable ones) as well as the analytical expression of the nonequilibrium potential. In this way we were able to obtain the transition rates and clearly quantify the SR phenomenon by means of the SNR.

We have seen that the nonhomogeneous spatial coupling, through density-dependent diffusivity, changes the effective dynamics of the system and, in agreement with [36], that such nonhomogeneous behavior could contribute to enhance the SR phenomenon. The form of the patterns, position of the attractors, and barrier's height explicitly depend on the noise intensity. We have found that there are ranges or windows of noise intensities where the phenomenon could arise (reentrance).

By considering the adiabatic limit and exploiting the twostate approximation we have theoretically predicted the occurrence of SR between those patterns. It is worth remarking here that it is the same noise source, the one that sustains the bistable dynamics and induces SR for transitions among the corresponding structures. The maximum of the SR response occurs in the symmetric case, in agreement with the results found in $[5,6]$. The SR phenomenon is robust with respect to variations of the $h$ parameter of diffusivity, and when $h$ decreases the SNR maximum increases and shifts toward higher $\lambda$ values. The last fact follows from the associated shift of the noise-induced transition to larger noise intensities which take place in the spatially uncoupled associated system [i.e., the zero-dimensional system resulting from suppressing the gradient term in Eq. (3)]. 
The consideration of more general forms of couplings in many component systems will allow us to analyze SR between noise-induced patterns in activator-inhibitor-like systems. We will also study, within the present framework, the competence between local and nonlocal spatial couplings $[4,6]$, etc. These aspects, together with Monte Carlo simulations of the different cases, will be the subject of further work.

\section{ACKNOWLEDGMENTS}

The authors thank Professor R. Toral for fruitful discussions. H.S.W. acknowledges partial support from ANPCyT, Argentina, and thanks the MECyD, Spain, for an award within the Sabbatical Program for Visiting Professors, and to the Universitat de les Illes Balears for the kind hospitality extended to him.
[1] L. Gammaitoni, P. Hänggi, P. Jung, and F. Marchesoni, Rev. Mod. Phys. 70, 223 (1998).

[2] J. F. Lindner, B. K. Meadows, W. L. Ditto, M. E. Inchiosa, and A. Bulsara, Phys. Rev. E 53, 2081 (1996).

[3] H. S. Wio, Phys. Rev. E 54, R3075 (1996); H. S. Wio and F. Castelpoggi, Proceedings of the Conference UPoN'96, edited by C. R. Doering, L. B. Kiss, and M. Schlesinger (World Scientific, Singapore, 1997); F. Castelpoggi and H. S. Wio, Europhys. Lett. 38, 91 (1997).

[4] F. Castelpoggi and H. S. Wio, Phys. Rev. E 57, 5112 (1998).

[5] S. Bouzat and H. S. Wio, Phys. Rev. E 59, 5142 (1999).

[6] H. S. Wio, S. Bouzat, and B. von Haeften, in Proceedings of the 21st IUPAP International Conference on Statistical Physics, STATPHYS21, edited by A. Robledo and M. Barbosa [Physica A 306C, 140 (2002)].

[7] W. Horsthemke and R. Lefever, Noise-Induced Transitions: Theory and Applications in Physics, Chemistry and Biology (Springer, Berlin, 1984).

[8] C. Van den Broeck, J. M. R. Parrondo, and R. Toral, Phys. Rev. Lett. 73, 3395 (1994); C. Van den Broeck, J. M. R. Parrondo, R. Toral, and R. Kawai, Phys. Rev. E 55, 4084 (1997).

[9] S. Mangioni, R. Deza, H. S. Wio, and R. Toral, Phys. Rev. Lett. 79, 2389 (1997); S. Mangioni, R. Deza, R. Toral, and H. S. Wio, Phys. Rev. E 61, 223 (2000).

[10] P. Reimann, Phys. Rep. 361, 57 (2002).

[11] R. D. Astumian and P. Hänggi, Phys. Today 55(11), 33 (2002).

[12] P. Reimann, R. Kawai, C. Van den Broeck, and P. Hänggi, Europhys. Lett. 45, 545 (1999).

[13] S. Mangioni, R. Deza, and H. S. Wio, Phys. Rev. E 63, 041115 (2001).

[14] J. García-Ojalvo, A. Hernández-Machado, and J. M. Sancho, Phys. Rev. Lett. 71, 1542 (1993).

[15] J. García-Ojalvo and J. M. Sancho, Noise in Spatially Extended Systems (Springer-Verlag, New York, 1999).

[16] B. von Haeften and G. Izús, Phys. Rev. E 67, 056207 (2003); G. Izús, P. Colet, M. San Miguel, and M. Santagiustina, ibid. 68, 036201 (2003).

[17] S. Mangioni and H. S. Wio, Phys. Rev. E 67, 056616 (2003).

[18] J. K. Douglas et al., Nature (London) 365, 337 (1993); J. J. Collins et al., ibid. 376, 236 (1995); S. M. Bezrukov and I. Vodyanoy, ibid. 378, 362 (1995).

[19] A. Guderian, G. Dechert, K. Zeyer, and F. Schneider, J. Phys. Chem. 100, 4437 (1996); A. Förster, M. Merget, and F. Schneider, ibid. 100, 4442 (1996); W. Hohmann, J. Müller, and F. W. Schneider, ibid. 100, 5388 (1996).
[20] A. Bulsara and G. Schmera, Phys. Rev. E 47, 3734 (1993); P. Jung, U. Behn, E. Pantazelou, and F. Moss, Phys. Rev. A 46, R1709 (1992); P. Jung and G. Mayer-Kress, Phys. Rev. Lett. 74, 2130 (1995); J. F. Lindner, B. K. Meadows, W. L. Ditto, M. E. Inchiosa, and A. Bulsara, ibid. 75, 3 (1995); F. Marchesoni, L. Gammaitoni, and A. Bulsara, ibid. 76, 2609 (1996).

[21] C. J. Tessone, H. S. Wio, and P. Hänggi, Phys. Rev. E 62, 4623 (2000).

[22] M. A. Fuentes, R. Toral, and H. S. Wio, Physica A 295, 114 (2001).

[23] R. Graham and T. Tel, in Instabilities and Non-equilibrium Structures III, edited by E. Tirapegui and W. Zeller (Kluwer, Dordrecht, 1991); H. S. Wio, in Fourth Granada Seminar in Computational Physics, edited by P. Garrido and J. Marro (Springer-Verlag, Berlin, 1997), p. 135.

[24] G. Izús et al., Phys. Rev. E 52, 129 (1995); G. Izús et al., Int. J. Mod. Phys. B 10, 1273 (1996); D. H. Zanette, H. S. Wio, and R. Deza, Phys. Rev. E 53, 353 (1996); F. Castelpoggi, H. S. Wio, and D. H. Zanette, Int. J. Mod. Phys. B 11, 1717 (1997).

[25] A. A. Zaikin, J. Kurths, and L. Schimansky-Geier, Phys. Rev. Lett. 85, 227 (2000); see also A. A. Zaikin, J. García-Ojalvo, R. Bascones, E. Ullner, and J. Kurths, Phys. Rev. Lett. 90, 030601 (2003), and references cited therein.

[26] M. Morillo, J. Gomez-Ordoñez, and J. M. Casado, Phys. Rev. E 52, 316 (1995).

[27] K. Kitara and M. Imada, Suppl. Prog. Theor. Phys. 64, 65 (1978).

[28] M. Ibañes, J. García-Ojalvo, R. Toral, and J. M. Sancho, Phys. Rev. Lett. 87, 020601 (2001).

[29] D. E. Strier, H. S. Wio, and D. H. Zanette, Physica A 226, 310 (1996).

[30] J. D. Murray, Mathematical Biology (Springer, Berlin, 1988).

[31] M. Doi and S. F. Edwards, The Theory of Polymer Dynamics (Clarendon, Oxford, 1986).

[32] Y. Fukai, The Metal-Hydrogen System (Springer, Berlin, 1993).

[33] B. McNamara and K. Wiesenfeld, Phys. Rev. A 39, 4854 (1989).

[34] P. Hänggi, P. Talkner, and M. Borkovec, Rev. Mod. Phys. 62, 251 (1990).

[35] For small $h, \lambda_{c}$ increases (and hence $\epsilon$ ) and Kramer's formulas are (in principle) no longer valid in this limit case.

[36] B. von Haeften, R. Deza, and H. S. Wio, Phys. Rev. Lett. 84, 404 (2000). 